\title{
Charge Carrier Dynamics upon Sub-bandgap Excitation in Methylammonium Lead lodide Thin Films: Effects of Urbach Tail, Deep Defects, and Two-Photon Absorption
}

\author{
Valentina M. Caselli, Zimu Wei, Marnix M. Ackermans, Eline M. Hutter, Bruno Ehrler, \\ and Tom J. Savenije*
}

Cite This: ACS Energy Lett. 2020, 5, 3821-3827

Read Online

\section{ACCESS | Llll Metrics \& More | 回 Article Recommendations | Sl Supporting Information}

ABSTRACT: To further understand the optoelectronic properties of metal halide perovskites, we investigate sub-bandgap absorption in methylammonium lead iodide $\left(\mathrm{MAPbI}_{3}\right)$ films. Charge carrier dynamics are studied using time-resolved microwave conductivity measurements using sub-bandgap excitation. From changes in the decay dynamics as a function of excitation energy and intensity, we have identified three regimes: (i) Band-like charge transport at photon energies above $1.48 \mathrm{eV}$; (ii) a transitional regime between 1.48 and $1.40 \mathrm{eV}$; and (iii) below $1.40 \mathrm{eV}$ localized optically active defects $\left(8 \times 10^{13} \mathrm{~cm}^{-3}\right)$ dominate the absorption at low intensities, while two-photon absorption is observed at high intensities. We determined an Urbach energy of approximately $11.3 \mathrm{meV}$, indicative of a low structural and/or thermal disorder. Surprisingly, even excitation $120 \mathrm{meV}$ below the bandgap leads to efficient charge transfer into electron (C60) or hole (spiro-OMeTAD) transport layers. Therefore, we conclude that for $\mathrm{MAPbI}_{3}$, the band tail states do not lead to nonradiative losses.

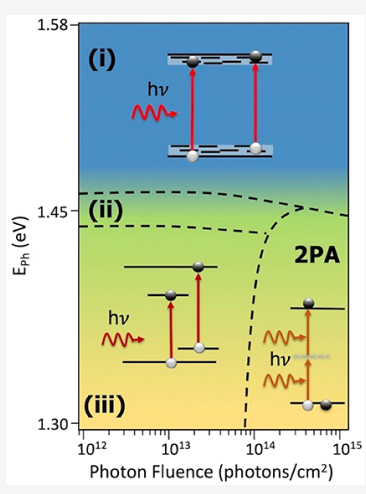

S ince the discovery that metal halide perovskites (MHPs) are excellent materials for optoelectronic applications, such as photovoltaics, ${ }^{1}$ the interest in these materials has grown rapidly. More recently, MHPs have been proven to be suitable candidates as active materials in photodetectors, ${ }^{2}$ light emitting diodes, ${ }^{3}$ X-ray detectors, ${ }^{4,5}$ and even nonlinear photonics. $^{6}$ Although these materials are typically produced via wet chemical techniques, semiconductors with a relatively low density of defects can be produced. However, to improve the efficiency of, for example, solar cells, it is important to identify states within the bandgap. Via those states, nonradiative recombination can occur, leading to a reduced open circuit voltage $\left(V_{\mathrm{OC}}\right)$. The electronic quality of a photoactive material for solar cell application is often assessed by comparing its bandgap with the obtainable voltage, i.e., by the bandgap-voltage offset $\left(E_{\mathrm{BG}} / q\right)-V_{\mathrm{OC}}{ }^{7}$ However, the absorption onset of a material is not always sharp due to the presence of defects. As such, an in depth study of its absorption edge and sub-bandgap absorption would lead to valuable knowledge to assess its suitability and potential for photovoltaic application.?

States in the bandgap are often classified as deep or shallow states, depending on whether trapped charges can be released by thermal energy or not. Experimental ${ }^{8-10}$ and in particular a huge amount of theoretical ${ }^{10-12}$ work has been devoted to the origin of these defects and their ability to act as trapping centers for excess charge carriers. Their impact on recombination is to a large extent related to the energy difference between the band edges and the energetic position of the defect state. ${ }^{13-18}$

In addition to trap states, tailing of the bands by the formation of an exponential distribution of states close to the valence band $(\mathrm{VB})$ maximum and conduction band (CB) minimum can occur. This leads to an exponential increase of the absorption just below the bandgap in semiconductor materials, which was described by Urbach in $1953 .{ }^{19}$ This distribution of states has been attributed to various types of lattice disorder. These can be structural, e.g., due to intrinsic defects or induced via doping or stoichiometric variations, compositional, e.g., atomic substitution, or thermal, e.g., lattice vibrations. ${ }^{20}$ If a material shows such Urbach tail, the spectral

Received: September 25, 2020

Accepted: November 4, 2020

Published: November 13, 2020

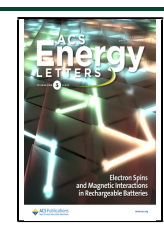



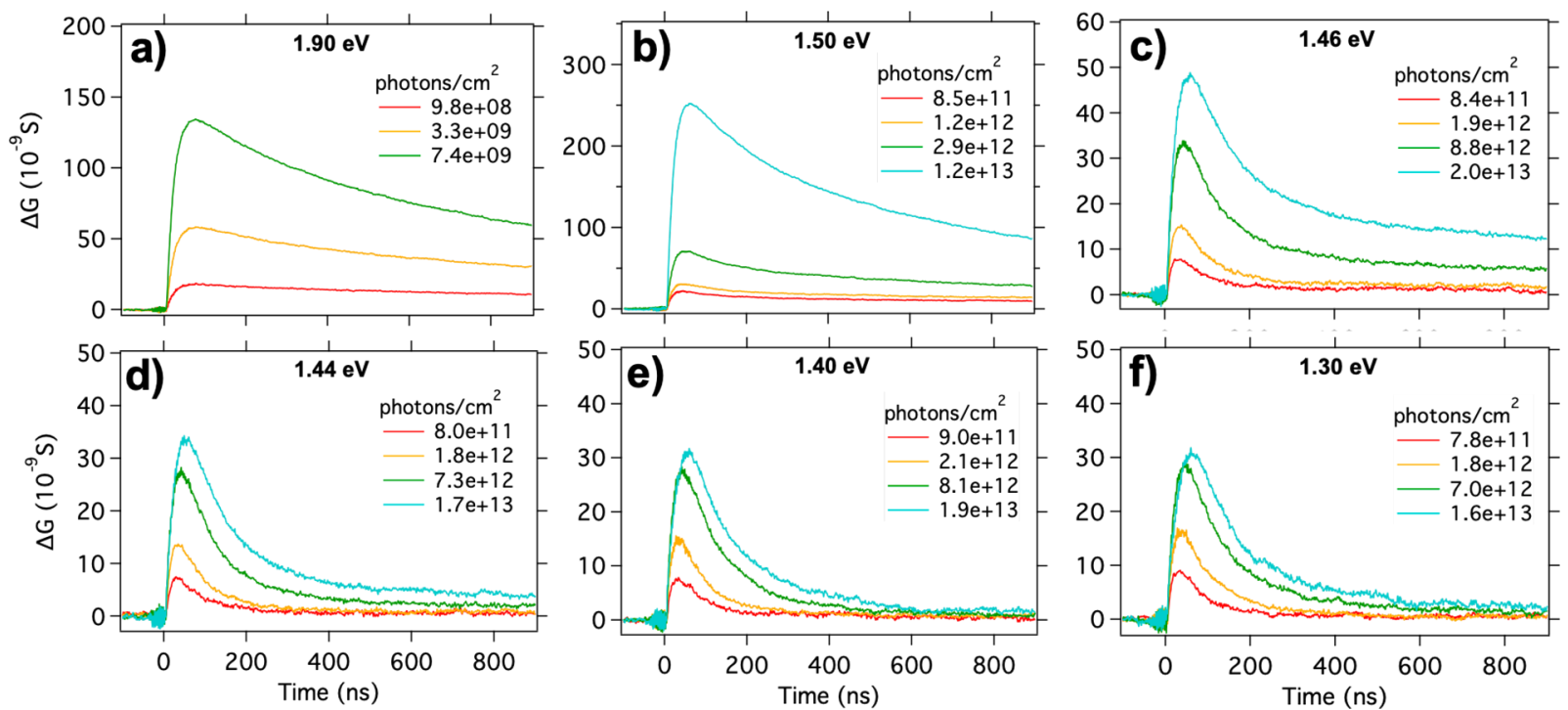

Figure 1. $\mathrm{MAPbI}_{3}$ thin film photoconductance signals for excitations at different photon energies and laser intensities. Note that from (a) above the bandgap to (b) below the bandgap, the photon fluence is more than 2 orders of magnitude higher.

and temperature dependence of its absorption coefficient, $\alpha$, below the bandgap can be expressed as

$$
\alpha(h \nu, T)=\alpha_{0} \exp \left[\frac{h \nu-E_{\mathrm{BG}}}{E_{\mathrm{U}}(T)}\right]
$$

where $\alpha_{0}$ is a constant, $E_{\mathrm{BG}}$ is the bandgap energy, and $E_{\mathrm{U}}$ is the Urbach energy. ${ }^{21}$ Previously, the temperature dependence of $E_{U}$ has been described by a summation of a temperatureindependent, static part, $E_{\mathrm{U}}(0)$, and a temperature-dependent part, defined as

$$
E_{\mathrm{U}}(T)=E_{\mathrm{U}}(0)+\frac{2 E_{\mathrm{U}}(0)}{e^{\Theta_{\mathrm{E}} / T}-1}
$$

in which $\Theta_{\mathrm{E}}$ is the Einstein phonon temperature. ${ }^{22}$ The $E_{\mathrm{U}}$ has been characterized for a variety of semiconductors, including $\mathrm{GaAs}^{23}$ as well as crystalline and amorphous $\mathrm{Si}^{24,25}$ For $\mathrm{MAPbI}_{3}$, the $E_{\mathrm{U}}$ has been determined by Fourier transform photocurrent spectroscopy (FTPS), ${ }^{22}$ photothermal deflection spectroscopy (PDS), 22,26 photoluminescence spectroscopy $(\mathrm{PL}){ }^{22,27}$ and external quantum efficiency (EQE) measurements. ${ }^{27}$ The values of $E_{\mathrm{U}}$ range between 14 and $25 \mathrm{meV}$, which can be attributed to the $\mathrm{MAPbI}_{3}$ sample quality and technique applied. Interestingly, Ledinsky et al. reported a very small value of $3.8 \mathrm{meV}$ for the static component and a total value of $14.3 \mathrm{meV}$ for $E_{\mathrm{U}}$ at room temperature. ${ }^{22}$

To investigate the behavior of carriers in $\mathrm{MAPbI}_{3}$ upon subbandgap excitation, we measured the photoconductance transients using the time-resolved microwave conductivity technique (TRMC). A central advantage of this technique is that we can record the charge carrier dynamics for different excitation wavelengths. Therefore, we can relate the energetic position of the state within the bandgap and the nature of the excited charge carrier. In contrast to FTPS and EQE, the TRMC technique is contactless; thus, unwanted contributions of the contacts or field interference to the photoconductivity signal are avoided. Contrarily to PL, but complementary to PDS, the TRMC technique allows us to investigate radiative and nonradiative decay kinetics or even electron transfer processes of the photoexcited charge carriers. The TRMC signal arises as a result of a change in microwave absorption due to the interaction of its electric field with the mobile charge carriers, i.e., free electrons and holes. ${ }^{28}$ The photoconductance signal is given by

$$
\Delta G(t)=e L \beta n \sum_{i} \mu_{i}
$$

in which $e$ is the elementary charge, $\beta$ is a geometrical factor, and $L$ is the sample thickness. From the sum of the mobilities, $\sum_{i} \mu_{i}$, of electrons and holes, the concentration, $n$, can be derived. As both charge carriers contribute to the measured photoconductance, from TRMC measurements, it is not possible to directly distinguish electron and hole signals. Additional information regarding the working principle of the TRMC technique can be found in the Supporting Information (SI).

From our study on the charge carrier dynamics induced by sub-bandgap excitation $\left(E_{\mathrm{Ph}}<E_{\mathrm{BG}}\right)$, we were able to identify two distinct regions for $\mathrm{MAPbI}_{3}$. In the first, ranging between $1.48 \mathrm{eV}<E_{\mathrm{Ph}}<E_{\mathrm{BG}}$, the carrier dynamics are identical to above-bandgap excitation. In this energy range, we observed an exponential increase of the absorption coefficient, $\alpha$, which can be related to the band tailing effect described by the Urbach rule. ${ }^{20}$ For energies between $1.40<E_{\mathrm{Ph}}<1.48 \mathrm{eV}$, a transition from band tailing to defect-related absorption is observed. At $E_{\mathrm{Ph}}<1.40 \mathrm{eV}$ and using low laser intensities, the photoexcitation and charge carrier dynamics are fully defect-related. On increasing the laser intensity, i.e., photon fluences $I_{0}>2 \times$ $10^{14}$ photons $/ \mathrm{cm}^{2}$ per pulse, two-photon absorption (2PA) occurs, as we have described recently. ${ }^{29}$ On deposition of an electron (C60)- or hole (spiro-OMeTAD)-selective transport layer on top of $\mathrm{MAPbI}_{3}$, we observe efficient charge transfer for photon energies as low as $1.44 \mathrm{eV}$. These results imply that for $\mathrm{MAPbI}_{3}$, excess carriers occupying the Urbach tail still contribute to photocurrent generation. ${ }^{27}$

To study the sub-bandgap absorption in $\mathrm{MAPbI}_{3}$ thin films, we first performed TRMC measurements at low intensities to avoid 2PA. ${ }^{29}$ The results for selected excitation energies are shown in Figure 1. On visible excitation at $1.90 \mathrm{eV}$, the photoconductivity shows a fast rise due to the formation of mobile carriers, followed by a decay due to charge 
recombination or trapping as discussed and modeled before. ${ }^{30}$ The initial magnitude scales with the excitation intensity, indicating that higher-order decay processes are not affecting the initial signal. Interestingly, on excitation at $1.50 \mathrm{eV}, 80$ meV below the bandgap $\left(E_{\mathrm{BG}}=1.58 \mathrm{eV}\right.$, as determined using a Tauc plot accounting for a direct allowed transition, Figure S2 in the SI), we observe similar charge carrier dynamics, with lifetimes in excess of multiple microseconds (compare Figure $1 \mathrm{a}$ and $\mathrm{lb}$ ). This implies that even at $1.50 \mathrm{eV}$, mobile electrons and holes can be generated. Note that the photon fluences as well as the $y$-axes used in Figure 1a,b are different because of the much higher absorption coefficient at $1.90 \mathrm{eV}$ compared to at $1.50 \mathrm{eV}$. More specifically, the laser intensities used for below-bandgap excitation (Figure $1 \mathrm{~b}$ ) are more than 2 orders of magnitude higher than for above the bandgap (Figure 1a). Nevertheless, the TRMC kinetics observed on excitation at 1.50 and $1.90 \mathrm{eV}$ are comparable, implying the same decay processes are operating on the excess charge carriers.

On excitation at 1.46 and $1.44 \mathrm{eV}$ (Figure 1c,d), a gradual transition can be observed. First of all, the TRMC signal size reduces. The substantial difference compared to the $1.50 \mathrm{eV}$ excitation can be related to a much lower absorption. Moreover, as the number of excess carriers is considerably reduced, the impact of charge carrier trapping on the kinetics becomes more pronounced. At low photon fluences, the charge carrier decay is less than 200 ns, while with higher intensities, we observe a gradual increase of the signal lifetime, which indicates that the fraction of long-lived mobile carriers increases.

For excitation energies far below the bandgap, the decay becomes faster, and the long-lived component is no longer present. Interestingly, for $E_{\mathrm{ph}}<1.40 \mathrm{eV}$, both magnitude and kinetics are the same at similar intensities (cf. Figure 1e,f). Moreover, it can be noted that the maximum signal is only linear at low intensities but reaches a plateau at approximately $2 \times 10^{13}$ photons $/ \mathrm{cm}^{2}$ per pulse. This indicates that there is a maximum number of possible excitations for the sub-bandgap linear absorption (SLA) process. These observations point to a generation mechanism related to the absorption from/to a finite number of localized defect states.

In previous work, we have performed an in-depth study of the 2PA process. Commonly, the 2PA process has been investigated by $Z$-scan measurements, both in single crystals and thin films. ${ }^{31-33}$ However, the accuracy of this technique in the quantification of the $2 \mathrm{PA}$ absorption coefficient, $\beta$, is affected by the influence of free carrier absorption on the measured transmitted light. By probing the carriers by microwaves, this issue is avoided. ${ }^{29}$ For the sample shown in Figure 2, we experimentally find that at $I_{0}>2 \times 10^{14}$ photons/ $\mathrm{cm}^{2}$, charge carriers can be generated by the simultaneous absorption of two photons $\left(E_{\mathrm{Ph}}=1.30 \mathrm{eV}\right)$. This process results in a gradual increase of the intensity-normalized photoconductance signal $\left(\Delta G /\left(\beta e I_{0}\right)\right)$ with intensity, as shown in Figure 2 for $E_{\mathrm{ph}}=1.30 \mathrm{eV}$. At this energy, we have previously determined a $\beta$ coefficient of approximately $11 \mathrm{~cm} /$ $\mathrm{MW}^{29}$ which implies that indeed above approximately $2 \times$ $10^{14}$ photons $/ \mathrm{cm}^{2}$, the $2 \mathrm{PA}$ process will start to dominate the photoconductance. Despite the low excitation energy, charges are now generated by the absorption of two photons; hence, the combined energy is sufficient for band-to-band excitation. Most importantly, this 2PA process results in charge carrier decay kinetics similar to those we observed in Figure 1a,b, i.e., for above and close to the bandgap excitation, respectively.

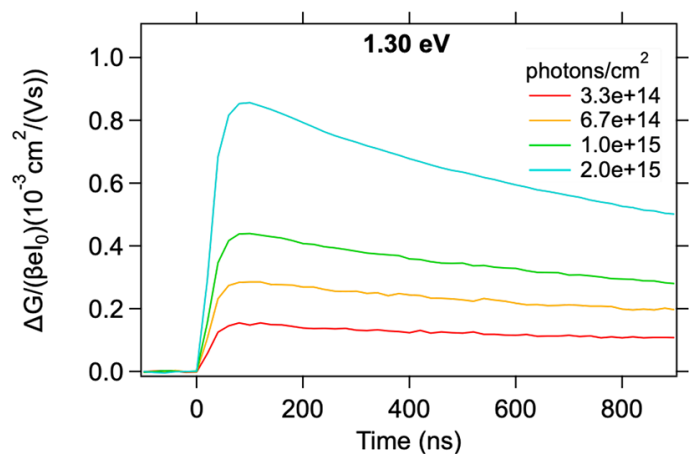

Figure 2. TRMC-intensity-normalized photoconductance signals of $\mathrm{MAPbI}_{3}$ thin film upon $1.30 \mathrm{eV}$ excitation at high laser intensities.

In order to examine if an excitation close to the bandgap is due to surface defects, samples have been analyzed before and after a light soaking treatment (LST), performed as described by Brenes et al. ${ }^{34}$ By this treatment, passivation of surface defect states occurs. ${ }^{34}$ As shown in Figure 3, the treated samples show an increase in lifetime to multiple microseconds, independent of the excitation energy $(1.90-1.45 \mathrm{eV})$. The improved lifetime indicates a reduced recombination rate, which can be related to the passivation of the surface states, as reported before. In contrast, the signal magnitude is not affected by the LST. From this observation, we conclude that the TRMC signal at $1.45 \mathrm{eV}$ is most likely due to sub-bandgap absorption in the bulk of the film, on which LST has no effect. If the absorption would originate from surface defects, passivation is expected to lower the height of the TRMC signal. The absence of any change in signal size hints again to a charge generation process in the bulk of the film. Moreover, this result confirms our previous conclusion that photoexcitations at this wavelength lead to band-like transport.

In order to obtain more knowledge on the nature of the excited carriers on sub-bandgap excitation, we extended our investigations to TRMC measurements on $\mathrm{MAPbI}_{3}$ samples covered with a thin, thermally evaporated C60 layer or a thin spin-coated 2,2',7,7'-tetrakis[N,N-di(4-methoxyphenyl)amino]-9,9'-spirobifluorene (spiro-OMeTAD) layer, acting as an electron- or hole-selective transport layer (ETL/HTL), respectively. As reported before, on optical excitation of such a bilayer system, CB electrons/VB holes can undergo electron/ hole transfer to the specific TL. ${ }^{28}$ Due to the relatively long response time of the TRMC measurement of $18 \mathrm{~ns}$, the charge transfer process itself cannot be revealed but only the result of the charge transfer. As detailed in the introduction, the observed TRMC signal is proportional to the product of the number of carriers and their mobility. Since the electron mobility in the organic layers is more than 1 order of magnitude lower than in the perovskite layer, ${ }^{35,36}$ the contribution of the transferred charges into the TLs to the total TRMC signal is small. Hence, the major part of the observed signal is due to the mobile charge carriers remaining in the perovskite layer. This implies that, provided charge transfer occurs, the TRMC signal of the bilayer is lower in magnitude as compared to the single perovskite layer. ${ }^{28,37} \mathrm{~A}$ TL also affects the decay kinetics: since the electrons and holes are physically separated after transfer, recombination is slowed down in the bilayer systems.

TRMC traces are shown in Figure 4 for both $\mathrm{MAPbI}_{3} / \mathrm{C} 60$ (Figure $4 \mathrm{a}, \mathrm{c}, \mathrm{e}$ ) and $\mathrm{MAPbI}_{3} /$ spiro-OMeTAD (Figure $4 \mathrm{~b}, \mathrm{~d}, \mathrm{f}$ ), 

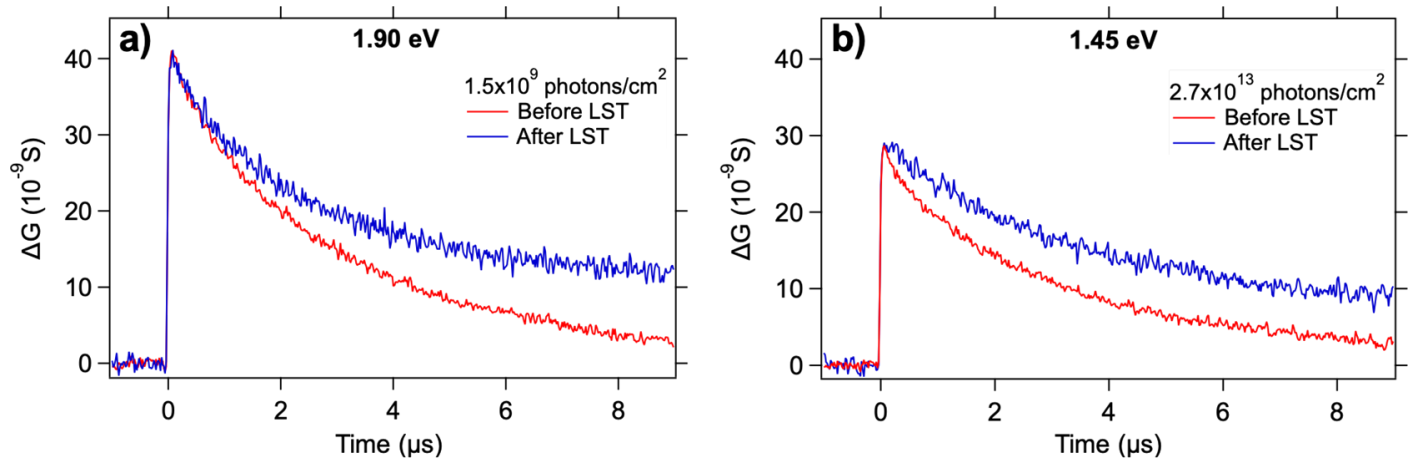

Figure 3. Photoconductance signal of $\mathrm{MAPbI}_{3}$ thin film before and after light soaking treatment (LST) for excitations of (a) $1.90 \mathrm{eV}$ at $1.5 \times$ $10^{9}$ photons $/ \mathrm{cm}^{2}$ and (b) $1.45 \mathrm{eV}$ at $2.7 \times 10^{13}$ photons $/ \mathrm{cm}^{2}$.
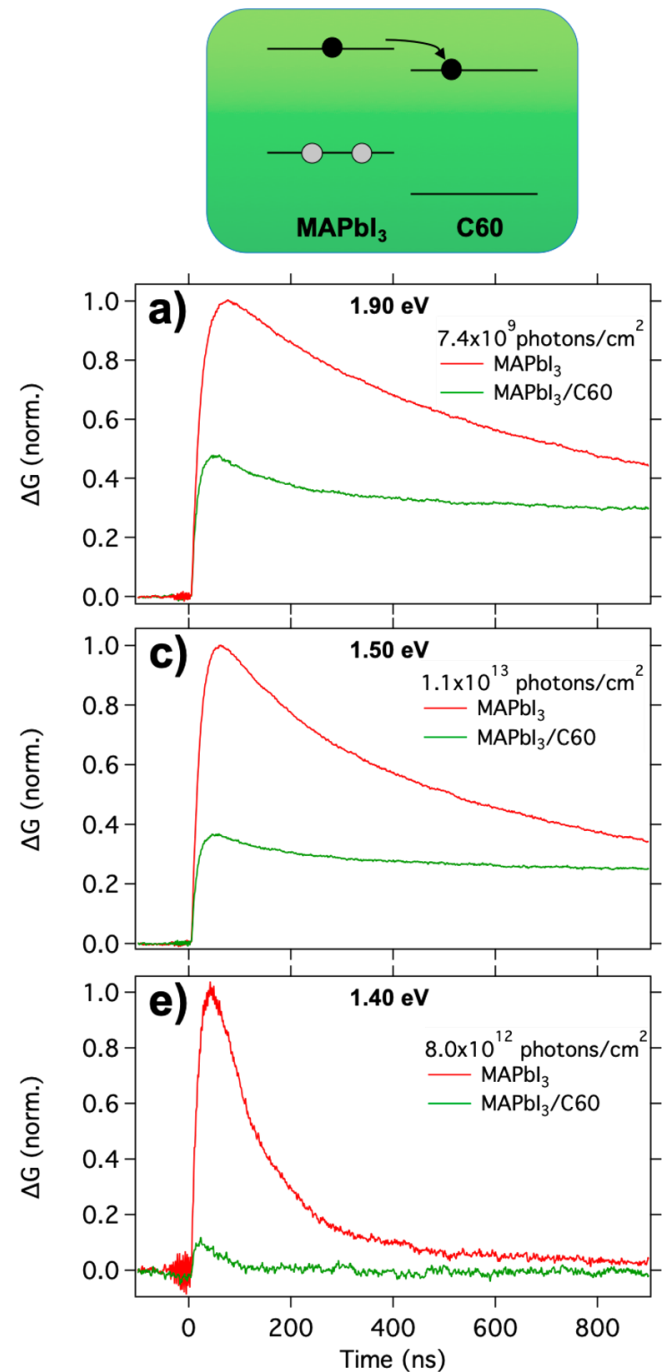
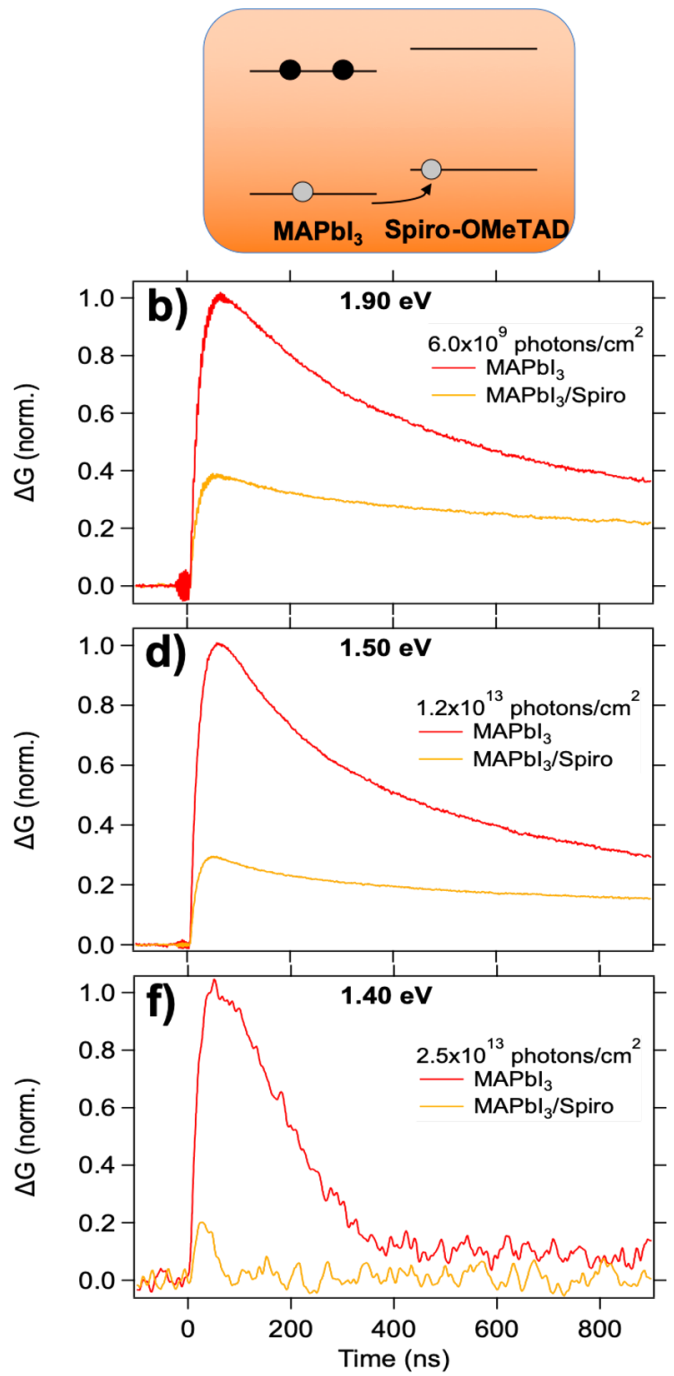

Figure 4. At the top, schematic representations of the charge transfer processes are shown for $\mathrm{MAPbI}_{3} / \mathrm{C} 60$ (left) and $\mathrm{MAPbI}_{3} / \mathrm{spiro}^{-}$ OMeTAD (right). The process occurs within the instrumental response time (18 ns), leading to the respective $\mathrm{MAPbI}_{3} / \mathrm{C} 60$ and $\mathrm{MAPbI}_{3} /$ spiro-OMeTAD bilayers' photoconductance signals upon front side excitation at $(\mathrm{a}, \mathrm{b}) E_{\mathrm{Ph}}=1.90 \mathrm{eV},(\mathrm{c}, \mathrm{d}) E_{\mathrm{Ph}}=1.50 \mathrm{eV}$, and $(\mathrm{e}, \mathrm{f}) E_{\mathrm{Ph}}=1.40$ eV.

and results are normalized for the maximum signal observed for the bare $\mathrm{MAPbI}_{3}$ layer. As shown in Figures $4 \mathrm{a}$,b, excitation at $1.90 \mathrm{eV}$ leads to very similar decay of the photoconductance signals upon changing from the neat $\mathrm{MAPbI}_{3}$ layer to a bilayer for both HTL and ETL, i.e., a clear reduction of the signal magnitude and elongation of the charge carrier lifetime. This indicates that both electron and hole transfer are efficient and that interfacial recombination occurs on longer time scales than the decay in the single layers. Most importantly, for $E_{\mathrm{ph}}=$ $1.50 \mathrm{eV}$ (Figure 4c,d) both electron and hole transfer are almost as efficient as for above-bandgap excitation. If the subbandgap excitation would involve optical excitation from or to 
a localized defect state, the immobile charge carrier could not contribute to the TRMC signal but also could not lead to charge transfer. In fact, only photoexcitations close to interface would lead to charge transfer. From our observation that charge transfer occurs efficiently, we conclude that for $E_{\mathrm{ph}}=$ $1.50 \mathrm{eV}$, photoexcited charges are thermally excited into the transport bands from where charge transport and subsequent transfer can occur efficiently. The efficient electron transfer to an ETL is in contrast to previous studies using spin-coated ETLs, ${ }^{38,28}$ in which either no transfer $^{38}$ or fast interfacial recombination $^{28}$ was observed. Vacuum deposition of C60 as used in this work seems to provide a more reliable method for efficient electron collection.

For excitation at $1.40 \mathrm{eV}$, the TRMC signals show a completely different behavior. For the bilayers (Figure 4e,f), we observe an almost complete disappearance of TRMC signal. Moreover, the lack of long-lived signals even at higher intensities indicates that no charge transfer has occurred. If either electrons or holes would have been transferred, we should have observed a marked difference in between the results of the two bilayers. This is due to the fact that, in at least one of the systems, one charge carrier would have remained in the $\mathrm{MAPbI}_{3}$ film, giving rise to a long-lived signal as observed in Figure 1a-d. From the heavily reduced signal magnitudes and enhanced decay on the introduction of the TLs, we might conclude that the charge carriers undergo fast interfacial trap-assisted recombination. However, this is far from likely in view of the efficient charge transfer observed on optical excitation at slightly higher energies. Therefore, we can conclude that, due to the introduction of the TL, the defect states are to some extent passivated and no longer optically active. This conclusion is in line with previous observations of surface passivation by C60 and analogues. 39,40

As introduced above, tailing of the bands can lead to an exponential absorption profile in the region just below the $E_{\mathrm{BG}}$. Here, we calculated the photon energy dependence of the absorption coefficient from TRMC results in the following way. From the measured $\Delta G$ and the known value for the mobility, the number of excitations, $n$, can be derived. Accounting for reflection losses, we can determine the effective photon fluence, $\left(I_{\text {eff }}\right)$, which after normalization yields the transmittance value, $T_{\text {TRMC }}$, defined by

$$
T_{\text {TRMC }}=\frac{I_{\text {eff }}-n L}{I_{\text {eff }}}
$$

Taking the negative natural logarithm of $T_{\text {TRMC }}$ and dividing by the thickness yields the absorption coefficient, $\alpha$. A more detailed description is given in the SI. The natural logarithm of $\alpha$ as a function of $E_{\mathrm{Ph}}$ is shown in Figure 5, together with the results of the PDS measurements. The TRMS and PDS measurements show excellent agreement for $E_{\mathrm{Ph}}>1.50 \mathrm{eV}$, which substantiates our assumption that the charge carrier generation yield and mobility for absorbed carriers in this energy range is constant, independent of the excitation energy.

As mentioned, at $E_{\mathrm{Ph}}<1.48 \mathrm{eV}$, the long-lived contribution to the TRMC signals becomes gradually smaller. By analysis of these TRMC traces, we are able to differentiate between charge carriers resulting from excitation into the band tail (red squares in Figure 5) and charge carriers excited to localized defect states (red circles in Figure 5). This differentiation has been accomplished by comparing the TRMC traces, with those recorded at $1.50 \mathrm{eV}$ (band-like transport) and at $1.40 \mathrm{eV}$

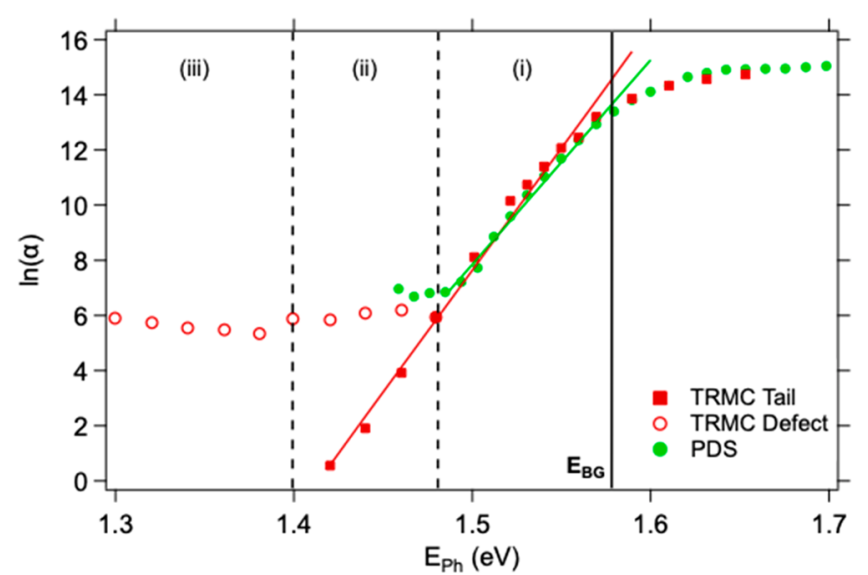

Figure 5. Logarithm of the absorption coefficient, $\alpha$, determined by TRMC as a function of photon energy. The solid black line corresponds to the bandgap energy, i.e., $1.58 \mathrm{eV}$ for the $\mathrm{MAPbI}_{3}$ film. The green and red fit lines have been used to determine the Urbach energy from PDS and TRMC measurements, respectively.

(excitation from/to defect state), as detailed in the SI. In this way, the value of $\alpha$ for $1.40<\mathrm{E}_{\mathrm{Ph}}<1.48 \mathrm{eV}$ can be determined more accurately, since we can account for the mobility of two (band-like transport) or one charge carrier. From the inverse of the slope of the straight line in the log-linear plot, an Urbach energy of approximately $16 \mathrm{meV}$ for $\mathrm{MAPbI}_{3}$ was found from PDS measurements, in agreement with previously reported values, $^{22,26,27}$ while $11.3 \mathrm{meV}$ was determined from the corrected tail contribution of the TRMC signals. As it has been observed, comparing FTPS and PL results, ${ }^{22}$ the sensitivity of the technique applied and the evaluation procedure are limiting factors in the determination of the Urbach energy in $\mathrm{MAPbI}_{3}$ thin films. Owning to the higher sensitivity of our TRMC measurements, we have been able to investigate the Urbach tail region down to its lowest limit, disentangling the tail states and defects absorption contributions.

Our measurements show that $\mathrm{MAPbI}_{3}$ has the following below-bandgap excitation regimes: (i) photoexcitation into the Urbach tail region $\left(1.48 \mathrm{eV}<E_{\mathrm{Ph}}<E_{\mathrm{BG}}\right.$ ) leads to charge carriers with band-like transport properties obeying the same decay processes as if they were the result of band-to-band excitation, (ii) the region of $1.40<\mathrm{E}_{\mathrm{Ph}}<1.48 \mathrm{eV}$ marks a transition from band tailing to trap-related absorption, (iii) excitation below $1.40 \mathrm{eV}$ is mainly due to absorption from/to a localized defect state.

The absorption profile below $1.40 \mathrm{eV}$ is characterized by a small but constant absorption coefficient, due to a relatively small concentration of defect states, which can be optically accessed. Previous photoconductance measurements exhibited a ratio of more than 5 orders of magnitude between EQE values obtained on excitation above the bandgap and at 1.3 eV. ${ }^{27}$ From Figure 5, a much smaller ratio is found. This can be explained by the fact that excitations at $1.3 \mathrm{eV}$ are indeed transitions to localized states. The involved charges have very short diffusion lengths and hence contribute with only low yields to the EQE. Since the detection in TRMC is based on the interaction of charges with high-frequency microwaves, collection of charges by electrodes is not required, and hence, this issue is avoided. On the basis of the TRMC signals at different intensities, we expect that the upper limit of the concentration to be in the order of $8 \times 10^{13} \mathrm{~cm}^{-3}$. 
Interestingly, this number is very close to the concentration of deep trap states $\left(N_{\mathrm{T}}\right)$ we find on performing a global mathematical analysis on the TRMC traces recorded on above-bandgap excitation, as described previously by Hutter et al. ${ }^{41}$ In this model (see SI, Figure S5), excess carriers decay by band-to-band recombination and in addition by deep trapping. By analyzing the traces at different intensities, an estimate for $N_{\mathrm{T}}$ can be derived (see SI, Figure S6). In view of the similarities of this number and the number of carriers as deduced from our sub-bandgap excitation measurements, we tend to conclude that these are the same defects. In view of the fast decay of these charges, these states are most likely detrimental for solar cell performance. Above $1.46 \mathrm{eV}$, the Urbach tail starts to dominate the absorption coefficient. Excitation into this tail yields charge carriers with similar dynamics as above-bandgap excitation, implying that charges excited into the tail are pushed toward the bands by thermal energy. These tail states are present within the bulk and cannot be affected by surface treatments. From the fact that efficient charge transfer toward both $\mathrm{C} 60$ and spiro-OMeTAD is observed, we conclude that the Urbach tail does not negatively affect the voltage or the photovoltaic performance of $\mathrm{MAPbI}_{3}$ based solar cells. Furthermore, the similar efficiencies for electron and hole transfer upon excitation in the Urbach tail indicates that the tailing of the VB and $\mathrm{CB}$ is rather symmetric.

Owing to its high sensitivity, the TRMC technique is demonstrated to be a valid alternative to commonly established methods to investigate the sub-bandgap absorption properties of thin films. On the basis of the changes in the charge carrier dynamics and consecutive charge transfer studies into C60 or spiro-OMeTAD, we were able to identify different subbandgap absorption regimes. We demonstrated that excitation into the Urbach tail leads to mobile carriers that can be efficiently transferred into the TLs. These charge carriers are thermally excited into the transport bands. Surface passivation by a light soaking treatment did not affect the magnitude of the signals, but it improved the carrier lifetime. As such, we believe that band tail states cannot be considered as detrimental for photovoltaic performance. In contrast, absorption from/to states below the Urbach tail leads to immobile, quickly decaying charge carriers. This observation hints toward a defect-related absorption. On the basis of the TRMC results, we can estimate the deep defect concentration to be in the order of $8 \times 10^{13} \mathrm{~cm}^{-3}$. This study provides a comprehensive overview of the generation and recombination processes in $\mathrm{MAPbI}_{3}$ thin films and opens the path to revealing the effective role of lattice vibrations and defects on charge carrier dynamics.

\section{ASSOCIATED CONTENT}

\section{SI Supporting Information}

The Supporting Information is available free of charge at https://pubs.acs.org/doi/10.1021/acsenergylett.0c02067.

Sample preparation; optical and structural analyses; a detailed description of the TRMC technique; description of kinetic model, detailed evaluation procedure of the sub-bandgap absorption coefficient, and PDS analysis details (PDF)

\section{AUTHOR INFORMATION}

\section{Corresponding Author}

Tom J. Savenije - Department of Chemical Engineering, Delft University of Technology, $2629 \mathrm{HZ}$ Delft, The Netherlands; 다이.org/0000-0003-1435-9885; Email: T.J.Savenije@ tudelft.nl

\section{Authors}

Valentina M. Caselli - Department of Chemical Engineering, Delft University of Technology, $2629 \mathrm{HZ}$ Delft, The Netherlands; 10 orcid.org/0000-0002-3730-5241

Zimu Wei - Department of Chemical Engineering, Delft University of Technology, $2629 \mathrm{HZ}$ Delft, The Netherlands

Marnix M. Ackermans - Center for Nanophotonics, NWOInstitute AMOLF, 1098 XG Amsterdam, The Netherlands

Eline M. Hutter - Center for Nanophotonics, NWO-Institute AMOLF, 1098 XG Amsterdam, The Netherlands; Department of Chemistry, Utrecht University, $3584 \mathrm{CB}$

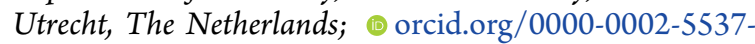
6545

Bruno Ehrler - Center for Nanophotonics, NWO-Institute AMOLF, 1098 XG Amsterdam, The Netherlands; (1) orcid.org/0000-0002-5307-3241

Complete contact information is available at:

https://pubs.acs.org/10.1021/acsenergylett.0c02067

\section{Notes}

The authors declare no competing financial interest.

\section{ACKNOWLEDGMENTS}

V.M.C. and T.J.S. received funding from the Dutch Research Council (NWO) Grant Number 739.017.004. The work of M.M.A., E.M.H., and B.E. is part of the research program of the Dutch Research Council (NWO) grant number VI.Veni.192.034.

\section{REFERENCES}

(1) Kojima, A.; Teshima, K.; Shirai, Y.; Miyasaka, T. Organometal Halide Perovskites as Visible-Light Sensitizers for Photovoltaic Cells. J. Am. Chem. Soc. 2009, 131, 6050-6051.

(2) Li, C.; Wang, H.; Wang, F.; Li, T.; Xu, M.; Wang, H.; Wang, Z.; Zhan, X.; Hu, W.; Shen, L. Ultrafast and Broadband Photodetectors Based on a Perovskite/Organic Bulk Heterojunction for LargeDynamic-Range Imaging. Light: Sci. Appl. 2020, 9, 31.

(3) Zou, Y.; Yuan, Z.; Bai, S.; Gao, F.; Sun, B. Recent Progress toward Perovskite Light-Emitting Diodes with Enhanced Spectral and Operational Stability. Mater. Today Nano 2019, 5, 100028.

(4) Mescher, H.; Schackmar, F.; Eggers, H.; Abzieher, T.; Zuber, M.; Hamann, E.; Baumbach, T.; Richards, B. S.; Hernandez-Sosa, G.; Paetzold, U. W.; et al. Flexible Inkjet-Printed Triple Cation Perovskite X-Ray Detectors. ACS Appl. Mater. Interfaces 2020, 12, 15774-15784.

(5) Tsai, H.; Liu, F.; Shrestha, S.; Fernando, K.; Tretiak, S.; Scott, B.; Vo, D. T.; Strzalka, J.; Nie, W. A Sensitive and Robust Thin-Film xRay Detector Using 2D Layered Perovskite Diodes. Sci. Adv. 2020, 6, eaay0815.

(6) Ferrando, A.; Martínez Pastor, J. P.; Suárez, I. Toward Metal Halide Perovskite Nonlinear Photonics. J. Phys. Chem. Lett. 2018, 9, $5612-5623$.

(7) De Wolf, S.; Holovsky, J.; Moon, S. J.; Löper, P.; Niesen, B.; Ledinsky, M.; Haug, F. J.; Yum, J. H.; Ballif, C. Organometallic Halide Perovskites: Sharp Optical Absorption Edge and Its Relation to Photovoltaic Performance. J. Phys. Chem. Lett. 2014, 5, 1035-1039.

(8) Baumann, A.; Väth, S.; Rieder, P.; Heiber, M. C.; Tvingstedt, K.; Dyakonov, V. Identification of Trap States in Perovskite Solar Cells. J. Phys. Chem. Lett. 2015, 6, 2350-2354. 
(9) Motti, S. G.; Gandini, M.; Barker, A. J.; Ball, J. M.; Srimath Kandada, A. R.; Petrozza, A. Photoinduced Emissive Trap States in Lead Halide Perovskite Semiconductors. ACS Energy Lett. 2016, 1, $726-730$.

(10) Meggiolaro, D.; Motti, S. G.; Mosconi, E.; Barker, A. J.; Ball, J.; Andrea Riccardo Perini, C.; Deschler, F.; Petrozza, A.; De Angelis, F. Iodine Chemistry Determines the Defect Tolerance of Lead-Halide Perovskites. Energy Environ. Sci. 2018, 11, 702-713.

(11) Yin, W.-J.; Shi, T.; Yan, Y. Unusual Defect Physics in CH3NH3PbI3 Perovskite Solar Cell Absorber. Appl. Phys. Lett. 2014, 104, 063903.

(12) Kim, J.; Lee, S. H.; Lee, J. H.; Hong, K. H. The Role of Intrinsic Defects in Methylammonium Lead Iodide Perovskite. J. Phys. Chem. Lett. 2014, 5, 1312-1317.

(13) Kirchartz, T.; Krückemeier, L.; Unger, E. L. Research Update: Recombination and Open-Circuit Voltage in Lead-Halide Perovskites. APL Mater. 2018, 6, 100702.

(14) Das, B.; Aguilera, I.; Rau, U.; Kirchartz, T. What Is a Deep Defect? Combining Shockley-Read-Hall Statistics with Multiphonon Recombination Theory. Phys. Rev. Mater. 2020, 4, 024602.

(15) Jin, H.; Debroye, E.; Keshavarz, M.; Scheblykin, I. G.; Roeffaers, M. B. J.; Hofkens, J.; Steele, J. A. It's a Trap! On the Nature of Localised States and Charge Trapping in Lead Halide Perovskites. Mater. Horiz. 2020, 7, 397-410.

(16) Hall, R. N. Germanium Rectifier Characteristics. Phys. Rev. 1951, 83, 228.

(17) Shockley, W.; Read, W. T. Statistics of the Recombinations of Holes and Electrons. Phys. Rev. 1952, 87, 835-842.

(18) Motti, S. G.; Meggiolaro, D.; Martani, S.; Sorrentino, R.; Barker, A. J.; De Angelis, F.; Petrozza, A. Defect Activity in Lead Halide Perovskites. Adv. Mater. 2019, 31, 1901183.

(19) Urbach, F. The Long-Wavelength Edge of Photographic Sensitivity and of the Electronic Absorption of Solids [8]. Phys. Rev. 1953, 92, 1324.

(20) Studenyak, I.; Kranjec, M.; Kurik, M. Urbach Rule in Solid State Physics. Int. J. Opt. Appl. 2014, 4, 76-83.

(21) Baník, I.; Kubliha, M.; Labaš, V.; Lukovičová, J.; Pavlendová, G.; Sín, P. Order and Disorder and Their Influences on Optical Absorption of Glasses in the Gap Region. AIP Conf. Proc. 2016, 1752, 040001.

(22) Ledinsky, M.; Schönfeldová, T.; Holovský, J.; Aydin, E.; Hájková, Z.; Landová, L.; Neyková, N.; Fejfar, A.; De Wolf, S. Temperature Dependence of the Urbach Energy in Lead Iodide Perovskites. J. Phys. Chem. Lett. 2019, 10, 1368-1373.

(23) Johnson, S. R.; Tiedje, T. Temperature Dependence of the Urbach Edge in GaAs. J. Appl. Phys. 1995, 78, 5609-5613.

(24) Cody, G. D. Urbach Edge of Crystalline and Amorphous Silicon: A Personal Review. J. Non-Cryst. Solids 1992, 141, 3-15.

(25) Cody, G. D.; Tiedje, T.; Abeles, B.; Brooks, B.; Goldstein, Y. Disorder and the Optical-Absorption Edge of Hydrogenated Amorphous Silicon. Phys. Rev. Lett. 1981, 47, 1480-1483.

(26) Sadhanala, A.; Deschler, F.; Thomas, T. H.; Dutton, S. E.; Goedel, K. C.; Hanusch, F. C.; Lai, M. L.; Steiner, U.; Bein, T.; Docampo, P.; et al. Preparation of Single-Phase Films of CH3NH3Pb(I 1- XBrx)3 with Sharp Optical Band Edges. J. Phys. Chem. Lett. 2014, 5, 2501-2505.

(27) Sutter-Fella, C. M.; Miller, D. W.; Ngo, Q. P.; Roe, E. T.; Toma, F. M.; Sharp, I. D.; Lonergan, M. C.; Javey, A. Band Tailing and Deep Defect States in $\mathrm{CH} 3 \mathrm{NH} 3 \mathrm{~Pb}(\mathrm{I} 1-\mathrm{XBrx}) 3$ Perovskites as Revealed by Sub-Bandgap Photocurrent. ACS Energy Lett. 2017, 2, 709-715.

(28) Hutter, E. M.; Hofman, J. J.; Petrus, M. L.; Moes, M.; Abellón, R. D.; Docampo, P.; Savenije, T. J. Charge Transfer from Methylammonium Lead Iodide Perovskite to Organic Transport Materials: Efficiencies, Transfer Rates, and Interfacial Recombination. Adv. Energy Mater. 2017, 7, 1602349.

(29) Wei, Z.; Guo, D.; Thieme, J.; Katan, C.; Caselli, V. M.; Even, J.; Savenije, T. J. The Importance of Relativistic Effects on Two-Photon
Absorption Spectra in Metal Halide Perovskites. Nat. Commun. 2019, 10,5342 .

(30) Savenije, T. J.; Guo, D.; Caselli, V. M.; Hutter, E. M. Quantifying Charge-Carrier Mobilities and Recombination Rates in Metal Halide Perovskites from Time-Resolved Microwave Photoconductivity Measurements. Adv. Energy Mater. 2020, 10, 1903788.

(31) Zhang, R.; Fan, J.; Zhang, X.; Yu, H.; Zhang, H.; Mai, Y.; Xu, T.; Wang, J.; Snaith, H. J. Nonlinear Optical Response of OrganicInorganic Halide Perovskites. ACS Photonics 2016, 3, 371-377.

(32) Kalanoor, B. S.; Gouda, L.; Gottesman, R.; Tirosh, S.; Haltzi, E.; Zaban, A.; Tischler, Y. R. Third-Order Optical Nonlinearities in Organometallic Methylammonium Lead Iodide Perovskite Thin Films. ACS Photonics 2016, 3, 361-370.

(33) Saouma, F. O.; Park, D. Y.; Kim, S. H.; Jeong, M. S.; Jang, J. I. Multiphoton Absorption Coefficients of Organic-Inorganic Lead Halide Perovskites $\mathrm{CH} 3 \mathrm{NH} 3 \mathrm{PbX} 3(\mathrm{X}=\mathrm{Cl}, \mathrm{Br}, \mathrm{I})$ Single Crystals. Chem. Mater. 2017, 29, 6876-6882.

(34) Brenes, R.; Guo, D.; Osherov, A.; Noel, N. K.; Eames, C.; Hutter, E. M.; Pathak, S. K.; Niroui, F.; Friend, R. H.; Islam, M. S.; et al. Metal Halide Perovskite Polycrystalline Films Exhibiting Properties of Single Crystals. Joule 2017, 1, 155-167.

(35) MacKenzie, R. C. I.; Frost, J. M.; Nelson, J. A Numerical Study of Mobility in Thin Films of Fullerene Derivatives. J. Chem. Phys. 2010, 132, 064904.

(36) Tiazkis, R.; Paek, S.; Daskeviciene, M.; Malinauskas, T.; Saliba, M.; Nekrasovas, J.; Jankauskas, V.; Ahmad, S.; Getautis, V.; Khaja Nazeeruddin, M. Methoxydiphenylaminesubstituted Fluorene Derivatives as Hole Transporting Materials: Role of Molecular Interaction on Device Photovoltaic Performance. Sci. Rep. 2017, 7, 150.

(37) Bouduban, M. E. F.; Queloz, V. I. E.; Caselli, V. M.; Cho, K. T.; Kirmani, A. R.; Paek, S.; Roldan-Carmona, C.; Richter, L. J.; Moser, J. E.; Savenije, T. J.; et al. Crystal Orientation Drives the Interface Physics at Two/Three-Dimensional Hybrid Perovskites. J. Phys. Chem. Lett. 2019, 10, 5713-5720.

(38) Leijtens, T.; Eperon, G. E.; Barker, A. J.; Grancini, G.; Zhang, W.; Ball, J. M.; Kandada, A. R. S.; Snaith, H. J.; Petrozza, A. Carrier Trapping and Recombination: The Role of Defect Physics in Enhancing the Open Circuit Voltage of Metal Halide Perovskite Solar Cells. Energy Environ. Sci. 2016, 9, 3472-3481.

(39) Shao, Y.; Xiao, Z.; Bi, C.; Yuan, Y.; Huang, J. Origin and Elimination of Photocurrent Hysteresis by Fullerene Passivation in CH3NH3PbI3 Planar Heterojunction Solar Cells. Nat. Commun. 2014, 5, 5784.

(40) Chen, B.; Rudd, P. N.; Yang, S.; Yuan, Y.; Huang, J. Imperfections and Their Passivation in Halide Perovskite Solar Cells. Chem. Soc. Rev. 2019, 48, 3842-3867.

(41) Hutter, E. M.; Eperon, G. E.; Stranks, S. D.; Savenije, T. J. Charge Carriers in Planar and Meso-Structured Organic-Inorganic Perovskites: Mobilities, Lifetimes, and Concentrations of Trap States. J. Phys. Chem. Lett. 2015, 6, 3082-3090. 